\title{
The effects of early aggressive therapy in JIA: results of the TREAT study
}

\author{
Carol A Wallace ${ }^{1 *}$, Edward H Giannini ${ }^{4}$, Steven J Spalding ${ }^{5}$, Philip J Hashkes ${ }^{12}$, Kathleen M O'Neil ${ }^{9}$, Andrew S Zeft ${ }^{16}$, \\ Ilona S Szer ${ }^{10}$, Sarah M Ringold ${ }^{1}$, Hermine Brunner ${ }^{4}$, Laura E Schanberg ${ }^{6}$, Robert P Sundel ${ }^{3}$, Diana Milojevic ${ }^{15}$, \\ Marilynn G Punaro ${ }^{14}$, Peter Chira ${ }^{13}$, Beth S Gottlieb ${ }^{11}$, Gloria C Higgins ${ }^{8}$, Norman T Ilowite ${ }^{2}$, Yukiko Kimura”, \\ Bin Huang ${ }^{4}$, Daniel J Lovell ${ }^{4}$
}

From 2011 Pediatric Rheumatology Symposium sponsored by the American College of Rheumatology Miami, FL, USA. 2-5 June 2011

\section{Purpose}

Early aggressive therapy has been shown to result in superior outcomes in adults with RA, but evidence for a similar benefit has not been demonstrated in children with JIA. The objective was to determine if aggressive treatment initiated within the first 12 mos after onset of extended oligoarticular (e-oligo), or RF + or (-) polyarticular JIA (poly JIA) can induce inactive disease (ID) within 6 mos (primary endpoint) and clinical remission on medication (CRM: ID for 6 continuous mos on medication; exploratory endpoint) within $1 \mathrm{yr}$ of starting therapy.

\section{Methods}

TREAT was designed as a multi-centered, prospective, double blind, randomized, placebo controlled clinical trial in children aged 2 to 16 yrs with e-oligo or poly JIA of $\leq$ 12 mos duration. Participants were randomized 1:1 into 1 of 2 aggressive treatment arms: (Arm 1) MTX $0.5 \mathrm{mg} /$ $\mathrm{kg} / \mathrm{wk} \mathrm{SQ}$ (40 mg max), plus etanercept $0.8 \mathrm{mg} / \mathrm{kg} / \mathrm{wk}$ (50 $\mathrm{mg} \max$ ), plus prednisone $0.5 \mathrm{mg} / \mathrm{kg} / \mathrm{d}$ (60 $\mathrm{mg} \max )$ tapered to 0 by 17 wks or; (Arm 2) MTX (same dose as Arm 1) plus etanercept placebo, plus prednisolone placebo, then followed on protocol for up to 12 mos. After 4 mos on therapy participants who failed to achieve at least an ACR Pediatric 70 received open label etanercept, MTX, and prednisolone in the same doses as Arm 1. At 6 mos the participants who achieved ACR Pediatric 70 but failed to achieve ID received open label medication as in Arm 1. Efficacy analyses focused on the intent-to-treat approach.

${ }^{1}$ Children's Hospital and Regional Medical, Seattle, WA, USA

Full list of author information is available at the end of the article
Safety data were recorded for all participants who received at least one dose of medication.

\section{Results}

15 centers enrolled 85 participants (64 [75\%] female) with a median age of 11.1 yrs and disease duration of 4.1 mos. $70 \%$ were $\mathrm{ANA}+, 33 \% \mathrm{RF}+$, and $33 \%$ anti-CCP+. At baseline the median physician's global assessment of disease activity and parent global assessment of overall well-being were 7 and 5 respectively. There were no significant interArm differences in any of these variables. At baseline, Arm 2 had a statistically higher mean ESR ( 45 vs. 29 mm/ hr) and active joint count (25.5 vs. 18.9) compared to Arm 1. By 4 mos, 30 of 42 (71\%) participants in Arm 1 and 19 of 43 (44\%) in Arm 2 achieved an ACR Pediatric 70 (X2 = 6.5; $\mathrm{p}<.011)$. By 6 mos, 17 of $42(40 \%)$ of participants in Arm 1 achieved ID, compared to 10 of 43 (23\%) in Arm 2 $(\mathrm{X} 2=2.91 ; \mathrm{p}=.088)$. Although all $6 \mathrm{ACR}$ pediatric core set variables showed highly significant improvement from baseline by 6 mos in both Arms ( $<$.001), 5 of 6 core set variables showed statistically greater improvement in Arm 1 vs. Arm 2. By 12 mos, 12 (14\%) participants achieved CRM; 9 (21\%) had remained in Arm 1, and 3 (6\%) had remained in Arm 2 throughout the study ( $\mathrm{p}=0.0534)$. There were no significant inter-arm differences in the incidence of Grade 3 or higher adverse events, including infections requiring systemic therapy. There were 3 SAEs: pneumonia (Arm 1), psychosis (open label), and bacteremia with septic arthritis (open label). All resolved without sequelae.

\section{Conclusion}

Although this trial did not reach its primary endpoint, early aggressive therapy in this cohort of children with 
severe JIA and a high rate of RF positivity resulted in substantial proportions of participants achieving an ACR Pediatric 70 by 4 mos, ID by 6 mos, and CRM within 12 mos of treatment initiation.

\section{Disclosure}

Carol A. Wallace: Amgen Inc., 2; Edward H. Giannini: None; Steven J. Spalding: None; Philip J. Hashkes: None; Kathleen M. O'Neil: None; Andrew S. Zeft: None; Ilona S. Szer: None; Sarah M. Ringold: None; Hermine Brunner: None; Laura E. Schanberg: None; Robert P. Sundel: None; Diana Milojevic: None; Marilynn G. Punaro: None; Peter Chira: None; Beth S. Gottlieb: Pfizer Inc, 5; Gloria C. Higgins: None; Norman T. Ilowite: None; Yukiko Kimura: None; Bin Huang: Amgen Inc., 2; Daniel J. Lovell: Abbott Laboratories, 9, Amgen Inc., 5, Bristol-Myers Squibb, 9, Centocor, Inc., 9, Hoffmann-La Roche, Inc., 9, Novartis Pharmaceuticals Corporation, 9, Regeneron Pharmaceuticals, Inc., 9, UBC, 9.

\section{Author details}

${ }^{1}$ Children's Hospital and Regional Medical, Seattle, WA, USA. ${ }^{2}$ Children'sHospital Montefiore, Bronx, NY, USA. ${ }^{3}$ Childrens Hospital Medical Center, Boston, MA, USA. ${ }^{4}$ Cincinnati Children's Hospital Medical Center, Cincinnati, OH, USA. ${ }^{5}$ Cleveland Clinic, Cleveland, OH, USA. ${ }^{6}$ Duke University Medical Center, Durham, NC, USA. ${ }^{7}$ Hackensack University Medical Center, Hackensack, NJ, USA. ${ }^{8}$ Nationwide Childrens Hospital, Columbus, OH, USA. ${ }^{9}$ Oklahoma University Health Science Center, Oklahoma City, OK, USA. ${ }^{10}$ Rady Childrens Hospital San Diego, San Diego, CA, USA. " ${ }^{11}$ Schneider Children's Hospital, New Hyde Park, NY, USA. ${ }^{12}$ Shaare Zedek Medical Center, Jerusalem, Israel, USA. ${ }^{13}$ Stanford University School of Medicine, Stanford, CA, USA. ${ }^{14}$ Texas Scottish Rite Hospital, Dallas, TX, USA. ${ }^{15}$ UCSF, San Francisco, CA, USA. ${ }^{16}$ University of Utah, Salt Lake City, UT, USA.

Published: 13 July 2012 in JIA: results of the TREAT study. Pediatric Rheumatology 2012 10(Suppl 1):A56.

\section{Submit your next manuscript to BioMed Central} and take full advantage of:

- Convenient online submission

- Thorough peer review

- No space constraints or color figure charges

- Immediate publication on acceptance

- Inclusion in PubMed, CAS, Scopus and Google Scholar

- Research which is freely available for redistribution

Submit your manuscript at www.biomedcentral.com/submit 